\title{
Study on Swing Movable Teeth Drive with External Generating Wave Mode
}

\author{
Yali YI*, Yunfei GAO**, Lei HE***, Herong JIN**** \\ *Yanshan University, 066004 Qinhuangdao, PR China, E-mail: yiyali@ysu.edu.cn \\ **Yanshan University, 066004 Qinhuangdao,PR China, E-mail:2933210447@qq.com \\ ***Yanshan University, 066004 Qinhuangdao, PR China, E-mail: $949528822 @ q q . c o m$ \\ ****Yanshan University, 066004 Qinhuangdao,PR China,E-mail:ysujhr@ysu.edu.cn \\ crossref http://dx.doi.org/10.5755/j01.mech.25.3.23738
}

\section{Introduction}

The movable teeth drive employ a train of rolling bodies (balls or rollers) to convert the two coaxial speed and power $[1,2]$. They possess a large gear ratio because of the few teeth difference, and a high loading capacity due to the multiple contacts between the members. Because of omitting the Oldham mechanism which is essential for the conventional planetary gear drives with few teeth difference, they have more compact structure [3]. There are two kinds of the generating wave modes: the internal generating wave mode and external generating wave mode. The internal generating wave movable teeth dive with wave generator inside, very similar to harmonic drive, becomes the first choice for research certainly. The working principle of the external generating wave movable teeth drive is illustrated in Ref. [4]. Using this principle, many kinds of external generating wave movable teeth drives have been proposed and fabricated, some of them have been used in automotive machinery and robotic machines [5-7].

Very few researchers investigated the external generating wave movable teeth drives and only focused on the transmission principle and structure design [8]. With the wave generator outside and usually connected to the motor directly, the imbalance due to an eccentricity of external generating wave movable teeth drive is much greater than that of the internal generating wave movable teeth drive, which will bring unwanted vibration, noise and even destruction of structures. These disadvantages severely restrict the practical application of external generating wave movable teeth drives [9].

In the present work, we develop an external generating wave swing movable teeth drive with two teeth difference which can be integrated with pulley drive to form a pulley reducer, i.e. the whole external generating wave swing movable teeth drive is put inside the big belt pulley. Thus the deceleration output of big pulley is used as the input of the external wave generating swing movable teeth drive to prevent the high frequency vibration induced by high speed input. Meanwhile, the symmetry structure of two teeth difference drive can keep the balanced and steady rotation compared to one tooth difference drive which has eccentric input wave generator. As a result, the above shortcomings can be overcome. Moreover, the two drives are connected in series without increasing the overall dimensions. Consequently, this newly developed integrated pulley reducer can achieve a large transmission ratio which is the product of the two transmission ratios with a compact structure. Without competing with traditional gears over the whole range of reduction gears in general engineering application, this integrated pulley reducer may solve definite problems in the special fields of engineering practice. The potential application of this integrated pulley reducer can be in the beam pumping unit to solve the short service life of the pinion in the first stage reducer. The gear ratio of the two-stage reducer of the beam pumping unit is smaller without reducing the overall drive ratio.

According to Ref. [10], the movable teeth drive may be regarded as multiply repeated cam mechanism, that is, the forward and inverse cam mechanisms are connected in series. Generally, the wave generator is the input cam, the rollers or balls with the carrier are the followers, and the ring gear is the output cam. The profile generation of the wave generator and the ring gear may refer to the design of the cam profile. With this thought, many laws of motion have been investigated and applied on the profile generation of the movable teeth drive. Nam et al. proposed a thin platetype speed reducer using balls for robots, defined the trajectory of the ball using trigonometric functions and analyzed the contact force using numerical method and FEM analysis [11]. Similar to the above tooth profile, Bara slotted closed sinusoidal channel on the input and output cam to fabricate a frontal-sinusoidal transmission with balls [12]. Yi et al. developed a new movable tooth mechanism based on five polynomial curves to achieve any tooth-difference pure rolling drive and studied the meshing characteristics [13]. By using trochoidal curves as the tooth profile, Mizukami and Terada developed a movable pins input type trochoid gear reducer, investigated its motion principle and analyzed the pressure angle $[14,15]$. For the movable teeth drive studied in this paper, the dynamic characteristics are particularly important due to the external generating wave mode, so the influence of tooth profile on the dynamic characteristics should be taken into account. The motion law without shock becomes the first choice. Sinusoidal acceleration motion law (also known as cycloidal displacement motion law) has smooth acceleration and smooth jerk (no any abrupt changes), ideal for high speed motion [16]. As a speed reduction drive, there are no dwells in the full cycle motion, that is, only rise-fall (RF) motion program.

In this work, sinusoidal acceleration and quintic polynomial motion are selected as the motion law of the swing teeth, and then tooth profiles of the wave generator and the ring gear are deduced using polar complex vector approach. On this foundation, curvature of the ring gear and pressure angles of the drive are also conducted. Then, virtual prototype is developed to perform kinematic simulation by 
Adams. Finally, a physical prototype is manufactured according to the theoretical analysis results. The simulation results and prototype development verify the feasibility and correctness of the theoretical analysis.

\section{Geometric design of a drive}

\subsection{Structure of drive}

The structure of an external generating wave swing movable teeth drive is shown in Fig.1. In general, in order to realize speed reduction drive, the outer wave generator is the driving element, a family of swing teeth with the teeth number $Z g$ are pivoted uniformly to the separator which is rigidly connected with the gear casing, the ring gear is the driven element with the teeth number $Z k$. The speed ratio of the drive $i$ can be calculated through $i=Z k /(Z k-Z g)$ which uses the Willis method for the reversed mechanism. For the two teeth difference drive, $|Z k-Z g|=2$, thus could achieve dynamic balance compared to one teeth difference drive.

When the wave generator rotates, it provides the swing movable teeth with a radial displacement to push them back and forth. At the same time, because the separator is fixed, the swing movable teeth drive the ring gear to rotate. Therefore, the coaxial speed conversion can be achieved with the reduction ratio of $i$. Drive of this type can be considered as cam mechanisms with many oscillating followers (the swing movable teeth). All the swing movable teeth mesh with both the wave generator and the ring gear simultaneously, thus the multi-tooth meshing effect enable a higher stiffness. And free of the Oldham mechanism facilitate smaller axial dimension.

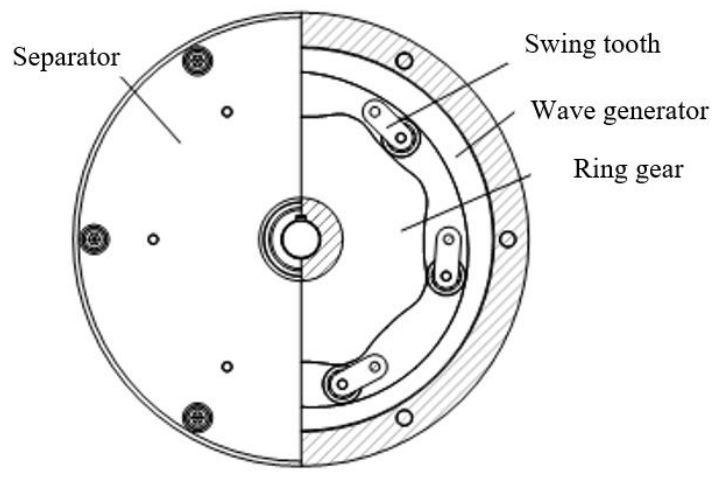

Fig. 1 Structure of a drive

\subsection{Tooth profile generation of the wave generator}

The overall mind map of the profile equation establishment was shown in Fig. 2 .

The basic form of the wave generator tooth profile will be set at first and followed by theoretical tooth profile establishment through the complex vector method. Then theoretical profile equation of the ring gear will be deduced based on the oscillating tooth drive theory. Finally, the actual profile equations of the wave generator and the ring gear can be obtained based on the isometric transformation theory of differential geometry.

Typically, in the movable teeth drives, whether they are external or internal generating wave mode, the tooth profile of the wave generator must be determined firstly, and then the tooth profile of the ring gear can be determined accordingly. As depicted in Fig. 3, starting from the base circle whose radius is $R$, the motion law of the radial dimension increment of the wave generator is set to be quintic polynomial in the actuating travel (from point $\mathrm{A}$ to point $\mathrm{B}$ ), and the motion law of the radial dimension decrement of the wave generator is set to be sinusoidal acceleration in the return travel (from point B to point C), which can be represented as:

$$
s(\varphi)=\left\{\begin{array}{ll}
10 h \frac{\varphi^{3}}{\phi^{3}}-15 h \frac{\varphi^{4}}{\phi^{4}}+6 h \frac{\varphi^{5}}{\phi^{5}}, & \left(0 \leq \varphi<\frac{\pi}{2}\right) \\
h\left[\frac{2 \phi-\varphi}{\phi}-\frac{1}{2 \pi} \sin \left(\frac{2 \pi}{\phi}(2 \phi-\varphi)\right)\right],\left(\frac{\pi}{2} \leq \varphi<\pi\right)
\end{array},\right.
$$

where: $h, \phi, \varphi$ represent the lift, motion angle for actuating travel, direction angle of the tooth profile vector of the wave generator, respectively. For the two teeth difference drive, $\phi=\pi / 2$.

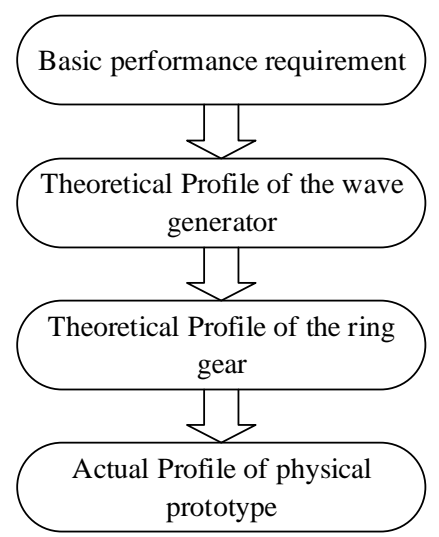

Fig. 2 The mind map of the profile equation establishment

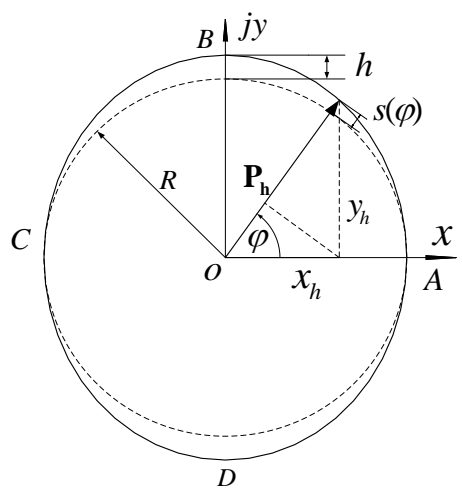

Fig. 3 Mathematical model for the generation of the wave generator tooth profile

Thus, a complete tooth profile $\mathrm{ABC}$ of the wave generator is constructed from point $\mathrm{A}$ to point $\mathrm{C}$. The tooth profile $\mathrm{CDA}$ and $\mathrm{ABC}$ are symmetry about origin $O$ to form the double phase tooth profile of external wave generator. The theoretical tooth profile of the wave generator can be obtained by Euler formula:

$$
\left\{\begin{array}{l}
p_{h}=R+s(\varphi) \\
\mathbf{P}_{\mathbf{h}}=p_{h} e^{j \varphi}=x_{h}+j y_{h}
\end{array},\right.
$$

where: $\mathbf{p}_{\mathbf{h}}, p_{h}$ represent the tooth profile vector of the wave generator and its modulus, respectively. Then the theoretical tooth profile of wave generator can be obtained as: 


$$
\left\{\begin{array}{l}
x_{h}=[R+s(\varphi)] \cos \varphi \\
y_{h}=[R+s(\varphi)] \sin \varphi
\end{array} .\right.
$$

By differentiating Eq.(3) with respect to $\varphi$, gives:

$$
\left\{\begin{array}{l}
\frac{d x_{h}}{d \varphi}=\dot{s}(\varphi) \cos \varphi-[R+s(\varphi)] \sin \varphi \\
\frac{d y_{h}}{d \varphi}=\dot{s}(\varphi) \sin \varphi+[R+s(\varphi)] \cos \varphi
\end{array} .\right.
$$

From the geometrical relationships shown in Fig.3, we obtain:

$$
\left\{\begin{array}{l}
\tan \varphi=\frac{y_{h}}{x_{h}} \\
p_{h}=x_{h} \cos \varphi+y_{h} \sin \varphi
\end{array} .\right.
$$

The derivation of $\dot{\mathbf{P}}_{\mathbf{h}}$ can be written as:

$$
\dot{\mathbf{P}}_{\mathbf{h}}=\dot{u} e^{j \lambda}=\dot{p}_{h} e^{j \varphi}+j \dot{\varphi} p_{h} e^{j \varphi}=\frac{d x_{h}}{d \varphi}+j \frac{d y_{h}}{d \varphi},
$$

where: $\dot{u}$ and $\lambda$ are the modulus of $\dot{\mathbf{P}}_{\mathbf{h}}$ and its direction.

Similar to the derivation of Eq. (5), yields:

$$
\left\{\begin{array}{l}
\tan \lambda=\frac{d y_{h}}{d \varphi} / \frac{d x_{h}}{d \varphi} \\
\dot{u}=\frac{d x_{h}}{d \varphi} \cos \lambda+\frac{d y_{h}}{d \varphi} \sin \lambda
\end{array} .\right.
$$

lowing:

Combining Eq. (4) with Eq. (7), we obtain the fol-

$$
\left\{\begin{array}{l}
\cos \lambda=\frac{\dot{s}(\varphi) \cos \varphi-[R+s(\varphi)] \sin \varphi}{\sqrt{\dot{s}(\varphi)^{2}+[R+s(\varphi)]^{2}}} \\
\sin \lambda=\frac{\dot{s}(\varphi) \sin \varphi+[R+s(\varphi)] \cos \varphi}{\sqrt{\dot{s}(\varphi)^{2}+[R+s(\varphi)]^{2}}}
\end{array} .\right.
$$

The theoretical tooth profile of the wave generator is offset $\mathbf{V}_{\mathbf{h}}$ to obtain its actual profile showed below, in which $\mathbf{V}_{\mathbf{h}}$ and $r$ represent the offset vector and its modulus:

$$
\mathbf{P}_{\mathbf{h}}{ }^{\prime}=\mathbf{P}_{\mathbf{h}}+\mathbf{V}_{\mathbf{h}}=\left(x_{h}-r \sin \lambda\right)+j\left(y_{h}+r \cos \lambda\right) .
$$

Substituting Eq. (8) and Eq. (9) into Eq. (4), the actual profile of the wave generator can be obtained as follows:

$$
\left\{\begin{array}{rl}
x_{h}{ }^{\prime} & =[R+s(\varphi)] \cos \varphi- \\
& -r \frac{\dot{s}(\varphi) \sin \varphi+[R+s(\varphi)] \cos \varphi}{\sqrt{\dot{s}(\varphi)^{2}+[R+s(\varphi)]^{2}}} \\
y_{h}{ }^{\prime} & =[R+s(\varphi)] \sin \varphi+ \\
+ & r \frac{\dot{s}(\varphi) \cos \varphi-[R+s(\varphi)] \sin \varphi}{\sqrt{\dot{s}(\varphi)^{2}+[R+s(\varphi)]^{2}}}
\end{array} .\right.
$$

\subsection{Tooth profile generation of the ring gear}

Before deriving the tooth profile equation of the ring gear, coordinate systems should be defined. According to the relative motion and the arrangement of the proposed drive, the coordinate systems for the generation of ring gear tooth profile is shown in Fig. 4. xoy is fixed to the separator, $x_{1} o y_{1}$ is fixed to the wave generator and $\boldsymbol{x}_{2} \boldsymbol{o y} \boldsymbol{y}_{2}$ is fixed to the ring gear. The three coordinates are coincided together in the beginning. From the theory of movable teeth drive, it can be obtained that $x_{2} o y_{2}$ will rotate $\theta_{i}=\theta / i$ when $x_{1} o y_{1}$ rotates $\theta$.

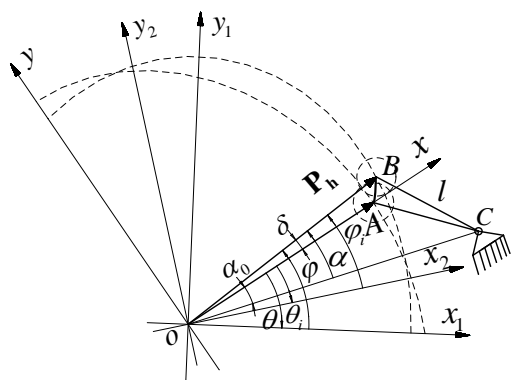

Fig. 4 Coordinate systems for the generation of ring gear tooth profile

The meanings of the symbols in Fig. 4 are shown in Table1.

Table1

The meanings of the symbols in Fig.4

\begin{tabular}{|c|c|}
\hline$\theta$ & The rotating angle of the wave generator \\
\hline$\theta_{i}$ & The rotating angle of the ring gear \\
\hline$\delta$ & The rotating angle of $\mathbf{P}_{\mathbf{h}}$ \\
\hline$\varphi$ & The rotating angle of $\mathbf{P}_{\mathbf{h}}$ relative to the wave generator \\
\hline$\varphi_{i}$ & The rotating angle of $\mathbf{P}_{\mathbf{h}}$ relative to the ring gear \\
\hline$C$ & The distance between point $\mathrm{O}$ and $\mathbf{C}$ \\
\hline$l$ & The length of the swing rod \\
\hline$\alpha$ & The angle between $\mathbf{P}_{\mathbf{h}}$ and OC at any time \\
\hline$\alpha_{0}$ & The angle between $\mathbf{P}_{\mathbf{h}}$ and OC in the beginning \\
\hline
\end{tabular}

According to cosine theorem, $\alpha_{0}$ and $\alpha$ can be determined as:

$$
\left\{\begin{array}{l}
\alpha_{0}=\operatorname{acos} \frac{R^{2}+C^{2}-l^{2}}{2 R C} \\
\alpha=\operatorname{acos} \frac{p(\varphi)^{2}+C^{2}-l^{2}}{2 p_{h} C}
\end{array}\right.
$$

From the geometric relationships shown in Fig.4, the relationship between angles can be written as:

$$
\left\{\begin{array}{l}
\delta=\alpha-\alpha_{0} \\
\theta=\varphi-\delta \\
\theta_{i}=\theta / i \\
\varphi_{i}=\left[\varphi+(1-i) \alpha_{0}+(i-1) \alpha\right] / i
\end{array} .\right.
$$

The tooth profile vector of the ring gear can be represented in the complex number form as: 


$$
\mathbf{P}_{\mathbf{k}}=p_{h} e^{j \varphi_{i}}=x_{k}+j y_{k} .
$$

In the $x_{2} \mathrm{Oy}_{2}$ coordinate system, the expanded form of the above equation can be written as follows to represent the theoretical tooth profile of the ring gear:

$$
\left\{\begin{array}{l}
x_{k}=[R+s(\varphi)] \cos \varphi_{i} \\
y_{k}=[R+s(\varphi)] \sin \varphi_{i}
\end{array} .\right.
$$
we obtain:

From the geometrical relationships shown in Fig.4,

$$
\left\{\begin{array}{l}
\tan \varphi_{i}=\frac{y_{k}}{x_{k}} \\
p_{h}=x_{k} \cos \varphi_{i}+y_{k} \sin \varphi_{i}
\end{array} .\right.
$$

The derivation of $P_{h}$ can be written as:

$$
\begin{aligned}
& \dot{\mathbf{P}}_{\mathbf{k}}=\dot{v} e^{j \mu}=\dot{p}_{h} e^{j \varphi_{i}}+j \dot{\varphi}_{i} p_{h} e^{j \varphi_{i}}= \\
& =\frac{d x_{k}}{d \varphi}+j \frac{d y_{k}}{d \varphi}
\end{aligned}
$$

where: $\dot{v}$ and $u$ are the modulus of $\dot{P}_{k}$ and its direction. $\dot{v}$ and $u$ can be determined using the following equation:

$$
\left\{\begin{array}{l}
\tan \mu=\frac{d y_{k}}{d \varphi} / \frac{d x_{k}}{d \varphi} \\
\dot{v}=\frac{d x_{k}}{d \varphi} \cos \mu+\frac{d y_{k}}{d \varphi} \sin \mu
\end{array} .\right.
$$

By differentiating Eq. (14) with respect to $\varphi$ ,yields:

$$
\left\{\begin{array}{l}
\frac{d x_{k}}{d \varphi}=\dot{s}(\varphi) \cos \varphi_{i}-[R+s(\varphi)] \dot{\varphi}_{i} \sin \varphi_{i} \\
\frac{d y_{k}}{d \varphi}=\dot{s}(\varphi) \sin \varphi_{i}+[R+s(\varphi)] \dot{\varphi}_{i} \cos \varphi_{i}
\end{array} .\right.
$$

From Eq. (17) and Eq. (18), gives:

$$
\left\{\begin{array}{c}
\cos \mu=\frac{\dot{s}(\varphi) \cos \varphi_{i}-[R+s(\varphi)] \dot{\varphi}_{i} \sin \varphi_{i}}{\sqrt{\dot{s}(\varphi)^{2}+\left\{[R+s(\varphi)] \dot{\varphi}_{i}\right\}^{2}}} \\
\sin \mu=\frac{\dot{s}(\varphi) \sin \varphi_{i}+[R+s(\varphi)] \dot{\varphi}_{i} \cos \varphi_{i}}{\sqrt{\dot{s}(\varphi)^{2}+\left\{[R+s(\varphi)] \dot{\varphi}_{i}\right\}^{2}}}
\end{array} .\right.
$$

The theoretical profile of the ring gear is offset $V_{k}$ to obtain its actual profile:

$$
\mathbf{P}_{\mathbf{k}}^{\prime}=\mathbf{P}_{\mathbf{k}}+\mathbf{V}_{\mathbf{k}}=\left(x_{k}-r \sin \mu\right)+j\left(y_{k}+r \cos \mu\right),
$$

where: $V_{k}$ and $r$ represent the offset vector and its modulus. Substituting Eq. (14) and Eq. (19) into Eq. (20), the actual profile of the ring gear can be expressed as:

$$
\left\{\begin{array}{rl}
x_{k}{ }^{\prime}= & {[R+s(\varphi)] \cos \varphi_{i}-} \\
- & r \frac{\dot{s}(\varphi) \sin \varphi_{i}+[R+s(\varphi)] \dot{\varphi}_{i} \cos \varphi_{i}}{\sqrt{[\dot{s}(\varphi)]^{2}+\left\{[R+s(\varphi)] \dot{\varphi}_{i}\right\}^{2}}} \\
y_{k}{ }^{\prime}= & {[R+s(\varphi)] \sin \varphi_{i}+} \\
+ & r \frac{\dot{s}(\varphi) \cos \varphi_{i}-[R+s(\varphi)] \dot{\varphi}_{i} \sin \varphi_{i}}{\sqrt{[\dot{s}(\varphi)]^{2}+\left\{[R+s(\varphi)] \dot{\varphi}_{i}\right\}^{2}}}
\end{array} .\right.
$$

\subsection{Ring gear curvature}

The curvature of a tooth profile is needed to avoid an undercutting. It can be known from differential geometry that the curvature of a curve can be calculated as follow:

$$
k=\frac{\left|\vec{r} \times \vec{r}^{\prime \prime}\right|}{|\vec{r}|^{3}} .
$$

The two-order derivative of the theoretical tooth profile of ring gear can be derived as:

$$
\begin{aligned}
& \ddot{\mathbf{P}}_{\mathbf{k}}=\ddot{v} e^{j \mu}+j \dot{v} \dot{\mu} e^{j \mu}= \\
& =\left\{\ddot{s}(\varphi)-[R+s(\varphi)]\left(\dot{\varphi}_{i}\right)^{2}+2 j \dot{\varphi}_{i} \dot{s}(\varphi)+\right. \\
& \left.+j \ddot{\varphi}_{i}[R+s(\varphi)]\right\} e^{j \varphi_{i}} .
\end{aligned}
$$

Substituting Eq. (16) and Eq. (23) into Eq. (22), yields the curvature of the ring gear tooth profile as follow:

$$
k=\frac{\dot{v} \dot{\mu}}{\dot{v}^{2}}
$$

in which:

$$
\left\{\begin{aligned}
\dot{v}^{2}= & \dot{s}(\varphi)^{2}+\left\{\dot{\varphi}_{i}[R+s(\varphi)]\right\}^{2} \\
\dot{\mu} \dot{v} & =\left\{\ddot{s}(\varphi)-[R+s(\varphi)] \dot{\varphi}_{i}^{2}\right\} \sin \left(\varphi_{i}-\mu\right)+ \\
& +\left\{2 \dot{s}(\varphi) \dot{\varphi}_{i}+\ddot{\varphi}_{i}[R+s(\varphi)]\right\} \cos \left(\varphi_{i}-\mu\right)
\end{aligned}\right.
$$

2.5. Research of pressure angle

Pressure angle is the angle at a meshing point between the lines of pressure (which is normal to the tooth surface) and the plane tangent to the meshing surface.

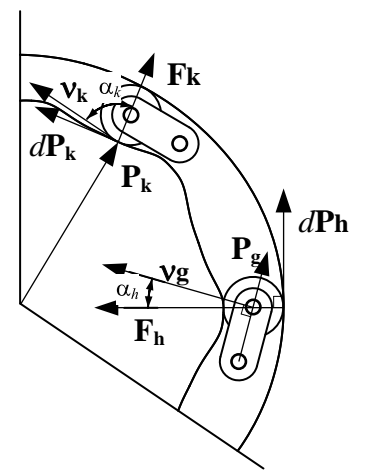

Fig. 5 Diagram of pressure angle analysis 
As shown in Fig.5, according to the definition of the pressure angle and the theory of movable teeth drive, the pressure angle between the wave generator and the movable teeth is $\alpha_{h}$, the pressure angle between the movable teeth and the ring gear is $\alpha_{k}$.

The pressure angle $\alpha_{h}$ can be represented as:

$$
\alpha_{h}=\arccos \frac{\mathbf{F}_{\mathbf{h}} \cdot \mathbf{v}_{g}}{\left|\mathbf{F}_{\mathbf{h}}\right| \cdot\left|\mathbf{v}_{g}\right|},
$$

where: $\mathbf{F}_{\mathbf{h}}, v_{g}$ represent the position vector of contact force between the wave generator and the movable teeth, the position vector of motion direction of the movable teeth, respectively.

From the geometrical relationships shown in Fig. $5, \mathbf{F}_{\mathbf{h}}, v_{g}$ can be obtained as follows:

$$
\left\{\begin{array}{l}
\mathbf{F}_{\mathbf{h}}=d \mathbf{P}_{h} e^{j \frac{\pi}{2}}=\dot{p}_{h} e^{j\left(\varphi+\frac{\pi}{2}\right)}+j p_{h} \dot{\varphi}_{i} e^{j\left(\varphi+\frac{\pi}{2}\right)} \\
\mathbf{v}_{\mathbf{g}}=\mathbf{P}_{g} e^{j \frac{\pi}{2}}=-p_{g} \sin \varphi+j p_{g} \cos \varphi
\end{array}\right.
$$

where: $d \mathbf{P}_{\mathbf{h}}$ is the tangential vector of the ring gear tooth profile. $d \mathbf{P}_{\mathbf{h}}$ is the position vector of the swing rod, $P_{h}$ is the modulus of $\mathbf{P}_{\mathbf{h}}$.

The solution method of the pressure angle $\alpha_{k}$ is the same as that of the pressure angle $\alpha_{h}$, it can be obtained as:

$$
\alpha_{k}=\arccos \frac{\mathbf{F}_{\mathbf{k}} \cdot v_{\mathbf{k}}}{\left|\mathbf{F}_{\mathbf{k}}\right| \cdot\left|v_{\mathbf{k}}\right|} .
$$

\section{Design examples}

In order to verify the correctness of the mathematical model of the drive proposed above, a computer program is developed to calculate the tooth profile data by Eqs. (3), (10), (14) and (21).

According to the speed ratio requirement of a transmission device the speed ratio of swing movable teeth drive was selected as 4 . Consequently, the teeth number $Z k$ of the ring gear is 8 , and the number $Z g$ of swing teeth is 6 . Choose SGM7J-06A type servo motor as the power input and its parameters are listed in Table 2.

Table2

Essential parameters of SGM7J-06A

\begin{tabular}{|c|c|}
\hline Model specification & SGM7J-06A \\
\hline Power & $0.6 \mathrm{~kW}$ \\
\hline Rotating speed & $3000 \mathrm{r} / \mathrm{min}$ \\
\hline Output torque & $1.91 \mathrm{~N} \cdot \mathrm{m}$ \\
\hline
\end{tabular}

The base radius and the lift can be chosen according to the output torque. Then the length of the swing rod and the radius of the roller can be determined by considering the non-interference conditions. The values of parameters are shown in Table 3.

According to the design parameters in Table 3, the tooth profiles of wave generator and ring gear are plotted through Matlab programing, shown in Fig.6. Curves 1 and 2 represent the theoretical tooth profiles of wave generator and ring gear respectively; curves 3 and 4 represent the actual tooth profile of the wave generator and the ring gear respectively. Fig. 7 shows the assembly of a drive via Solidworks.

Table3

Design parameters of a drive

\begin{tabular}{|c|c|}
\hline$R / \mathrm{mm}$ & 60 \\
\hline$C / \mathrm{mm}$ & 62 \\
\hline$l / \mathrm{mm}$ & 15 \\
\hline$h / \mathrm{mm}$ & 8 \\
\hline$r / \mathrm{mm}$ & 8 \\
\hline$Z_{k}$ & 8 \\
\hline$Z_{g}$ & 6 \\
\hline
\end{tabular}

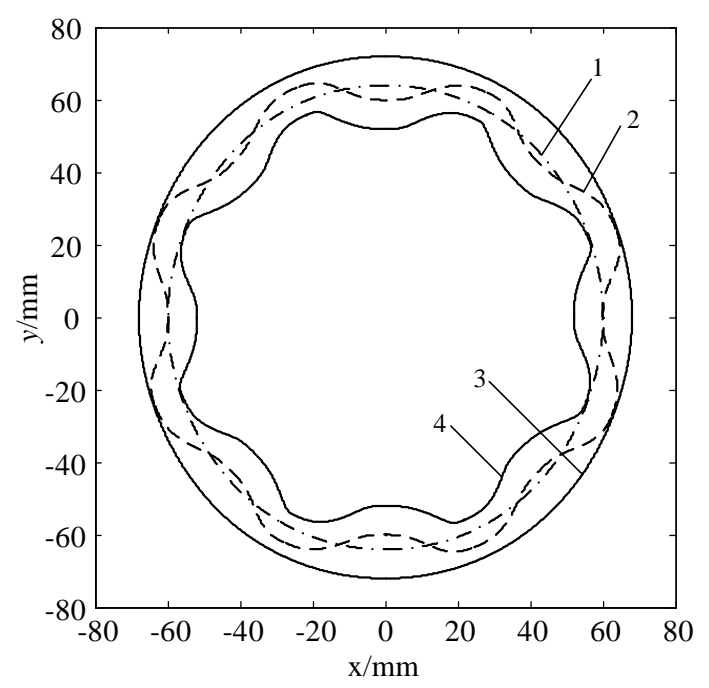

Fig. 6 Profile of wave generator and ring gear

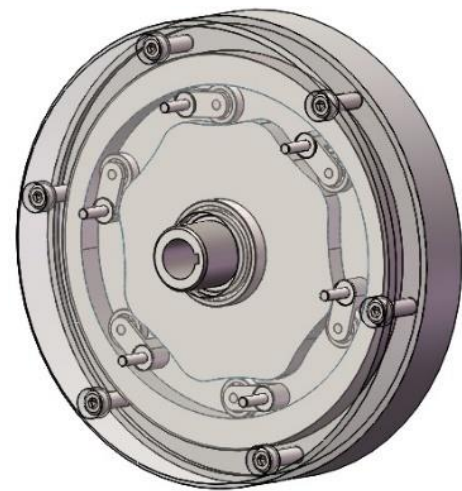

Fig. 7 Assembly of a drive

\subsection{Ring gear curvature calculation}

From Eq. (24) and Eq. (25), the curvature curve of the ring gear theoretical profile within one cycle is shown in Fig. 8 . The maximum curvature is 0.0543 , at which point the rotating angle of the ring gear is 17.4 degrees, and the corresponding curvature radius here is $18.42 \mathrm{~mm}$. The minimum curvature is -0.026 , at which point the rotating angle of the ring gear is 39.8 degrees, and the corresponding curvature radius here is $38.46 \mathrm{~mm}$. The actual tooth profile of the ring gear is the normal equidistant line of the theoretical tooth profile. In order to avoid undercutting, the radius of the movable teeth should be less than the minimum curvature radius of the ring gear theoretical tooth profile, that is, $r$ should be less than $18.42 \mathrm{~mm}$. 


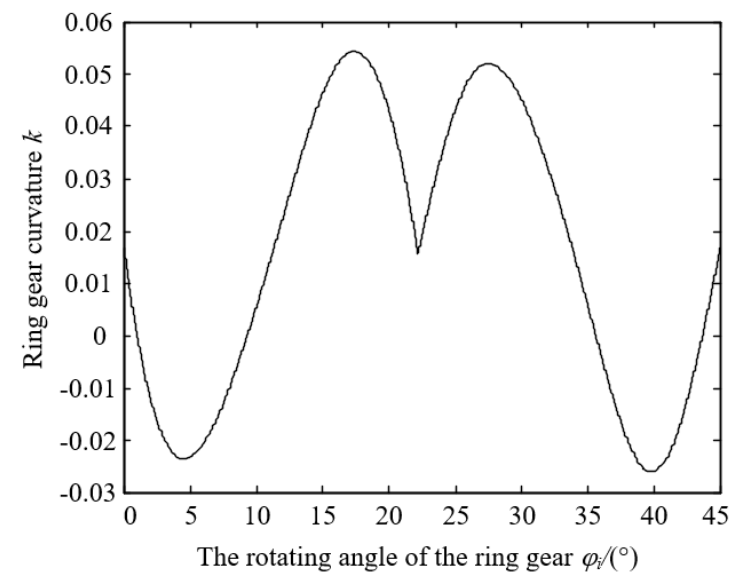

Fig. 8 Profile of wave generator and ring gear

\subsection{Pressure angle calculation}

According to Eq. (26) and Eq.(27), and using structural parameters given in Table 3 . The curve of the pressure angle $\alpha_{k}$ between the wave generator and the movable teeth is obtained as shown in Fig.9. The value of the pressure angle $\alpha_{k}$ is small from beginning to end and the maximum value is 31 degrees, which means nice driving effectiveness.

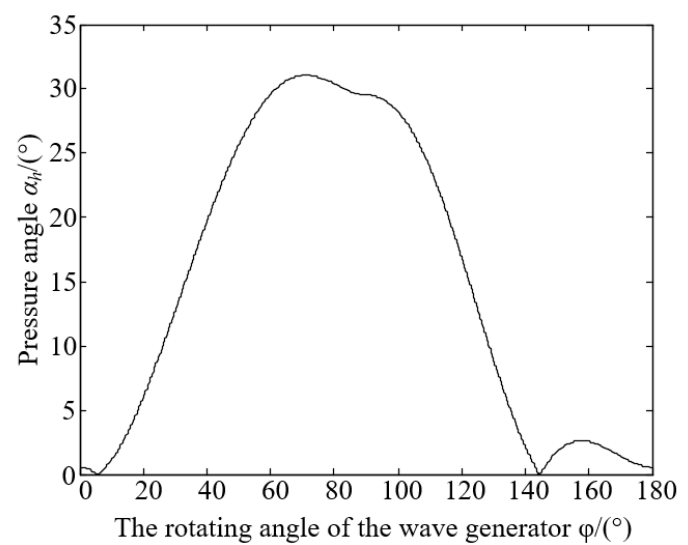

Fig. 9 Profile of wave generator and ring gear.

According to Eq. (28), the pressure angle between movable teeth and ring gear is calculated. As shown in Fig.10, when the rotating angle of the wave generator is 133.2 degrees, the pressure angle $\alpha_{k}$ is the minimum, which is 58.5 degrees.

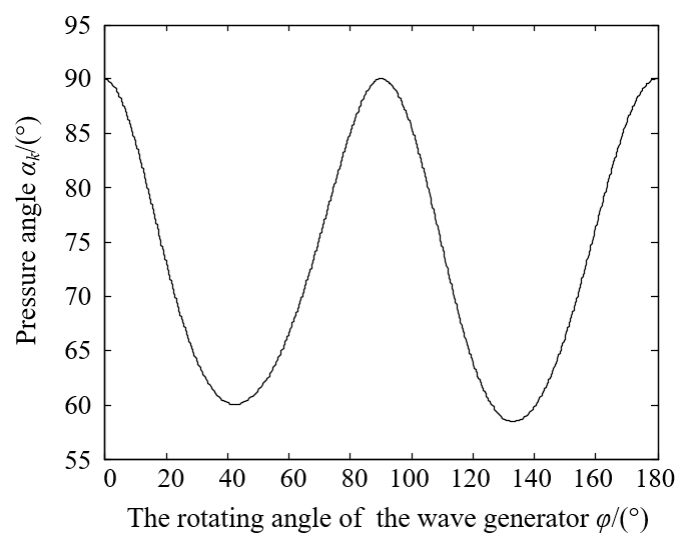

Fig. 10 The pressure angle between movable teeth and ring gear

\section{Kinematic simulation analysis}

The kinematic and dynamic analyses are implemented through Adams software to verify speed ratio and contact force of the drive. Through the verification, it can be seen that the kinematic properties of the virtual prototype are close to the theoretical value to verify the correctness of theoretical analysis. On the basis of the simulation, a solid prototype is manufactured with the same structural parameters.

Import the 3D model into Adams, define quality attributes and add corresponding constraints to get ready for later analysis. Then set the servo motor SGM7J-06A to be the power input, the parameters of which are listed in Table 2 . In the simulation process, there are 3000 steps during the simulating $0.1 \mathrm{~s}$ so that both precision and calculating efficiency can be taken into account at the same time. The final simulating results were shown below. Fig. 11 shows the instantaneous rotating speeds of the wave generator and the ring gear. It can be observed that the rotational speed of the output shaft sharply increased at first and then fluctuated at an average of $4488.4 \mathrm{deg} / \mathrm{s}$, which conformed to real work conditions. The theoretical output rotational speed was $4500 \%$ s when the input rotational speed of the wave generator was $18000^{\circ}$ s. And as shown in Fig.11, the deviation between simulation result and theoretical value was less than $5 \%$, which proved that the drive can achieve stable speed ratio. Fig. 12 shows the contact force between the ring gear and one of a swing tooth when being applied a resistance torque of $5.84 \mathrm{~N} \cdot \mathrm{m}$ during the gearing process.

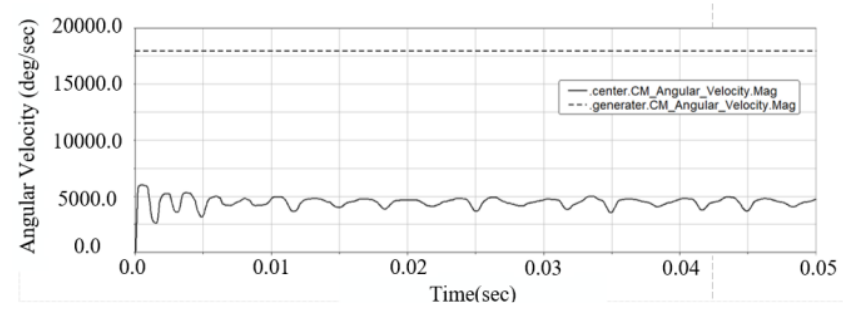

Fig. 11 Rotating speeds of the input and output components

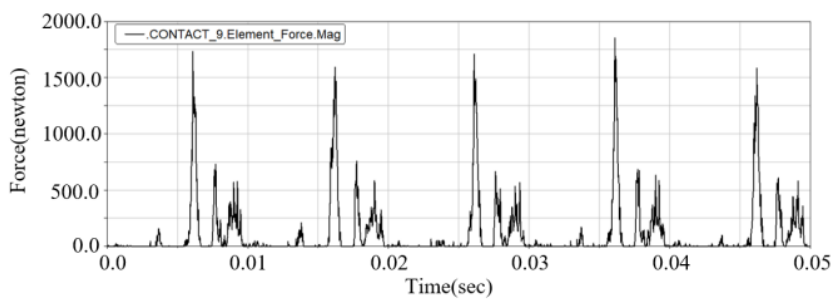

Fig. 12 Instantaneous contact force

From Fig. 12, it can be seen that the biggest contact force is $1853 \mathrm{~N}$ at the rise stage of the movable teeth motion process. And the contact force is close to $0 \mathrm{~N}$ at the return stage of the movable teeth motion process. Even though, the contact stress was still within the permissible range of the components' material, which provide a basic reference for future structural strength and output power designing at the same time. And the cyclic variation of the contact force coincided with the engagement period.

Considering the above design parameters, a physical prototype was manufactured, shown in Fig. 13. 
The prototype was installed on a test platform shown in Fig. 14 for running test. The coaxial speed reduction can be achieved well under dry friction condition. But impact and noise occurred during operation, which was mainly caused by manufacturing errors, assembly errors and dry friction condition. By adding grease, the impact and noise were greatly reduced, while the smoothness of the operation had been greatly improved.

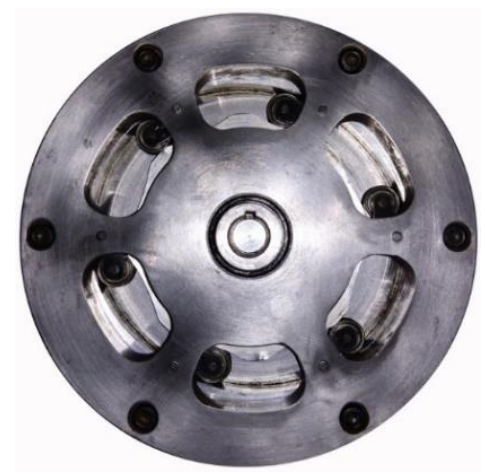

Fig. 13 Physical prototype of a drive

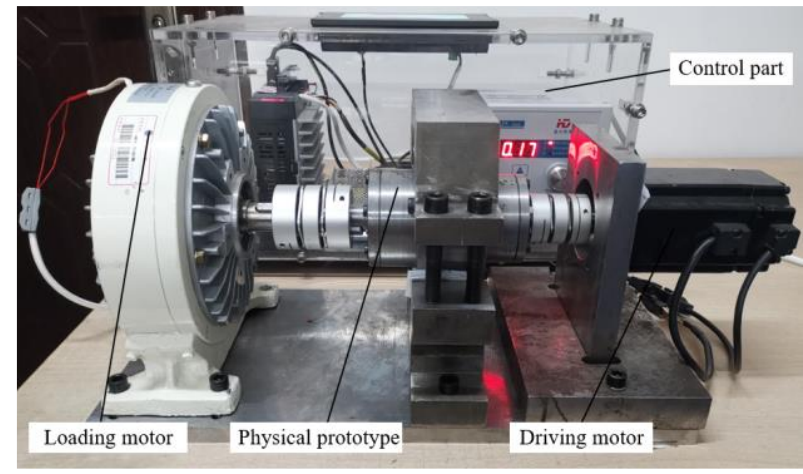

Fig. 14 Test run of a prototype

\section{Conclusions}

This study has proposed an external generated swing teeth speed reducer and the steps of parametric design of its tooth profile. The operating principle is illustrated. Curvature of the ring gear and pressure angles of the drive are analyzed. And a solid prototype is manufactured and tested. The results can be elaborated as follows:

1. The use of sinusoidal acceleration and quintic polynomial motion in the designing process of the tooth profile made it possible to obtain a higher stability. The characteristic of two-tooth difference made it easy to realize dynamic balance so that a high rotational speed became possible.

2. The rotational speed of the ring gear fluctuated at an average of $4488.4 \mathrm{deg} / \mathrm{s}$ when applied a rotating speed of $18000 \mathrm{deg} / \mathrm{s}$ on the wave generator. And the contact force of meshing pairs changed periodically with a period of $0.01 \mathrm{~s}$, in which the input shaft would rotate $180 \mathrm{deg}$.

3. Kinematic simulation verified the correctness of the tooth profile design. After a simple test, its operating characteristics were found well after being applied lubrication.

4. By integrating the proposed drive into pulley drive, it is possible to form a larger gear ratio reducer with more compact structure which has promising applications in the beam pumping unit or other similar equipment.

\section{Acknowledgment}

This project is supported by the National Natural Science Foundation of China (No. 51605416), Colleges and Universities in Hebei Province Science and Technology Research Youth Fund (No. QN2016095), and Independent Research Program of the Young Teachers of Yanshan University (No.15LGA004)

\section{References}

1. Qu, J. F. 1993. Theory of movable teeth drive, China Machine Press. I: 1-10 (in Chinese).

2. Liang, S. M. 2002. Study on elasto-dynamic model of swing movable teeth transmission system, Chinese Journal of Mechanical Engineering 38:142.

htps://doi.org/10.3901/jme.2002.supp.142.

3. Begljakov, V. Y.; Timofeev, V. Y.; Dokhnenko, M. V. 2014. Parameters of force interaction of elements the wave transmission with intermediate rolling bodies in Geokhod's transmission, Applied Mechanics and Materials 682282-287.

https://doi.org/10.4028/www.scientific.net/amm.682. 282.

4. Yi, Y, L.; Gao, Y, F.; Wei, R.; Jin, H. R.; 2019. Teeth profile parameter design and transmission property analysis of external generating wave movable teeth transmission, The Journal of Engineering 2019(3):299-305.

https://doi.org/10.1049/joe.2018.8957.

5. Ikan, A.; As, I.; Lazurkevich, A. V. 2016. Aspect of geometric calculation of the planetary gear train with intermediate rollers, IOP Conf. Series: Materials Science and Engineering 124(1): 1-5.

https://doi.org/10.1088/1757-899x/124/1/012003.

6. Ikan, A.; As, I.; Lazurkevich, A. V. 2016. Load analysis of the planetary gear train with intermediate rollers, IOP Conf. Series: Materials Science and Engineering 124(1): 1-6. https://doi.org/10.1088/1757-899x/124/1/012004.

7. Li, J. F.; He, A. Y.; Dong, X. R. 2011. Geometrical design of the movable rolling tooth transmission with cam actuating, Journal of Mechanical Engineering 47(1): 2429. https://doi.org/10.3901/jme.2011.01.024.

8. Belyev, A. 1986. Mechanical transmission with intermediate bodies increased accuracy and endurance, TPI Publishment (in Russian).

9. Plekhanov, M. S. 2013. Precision electromechanical drive for space systems, Tomsk Polytechnic University, C22:321-324.

10. Lustenkov, M. E 2010. Planetary ball transmissions strength calculation, Russian Engineering Research 30(9): 862-866. https://doi.org/10.3103/s1068798x10090030.

11. Nam, W. K.; Shin, J. W.; Oh, S. H. 2013. Design of thin plate-type speed reducers using balls for robots, Journal of Mechanical Science and Technology 27(2): 519-524. https://doi.org/10.1007/s12206-012-1242-5.

12. Bara, M. 2004. pects of frontal-sinusoidal transmission with balls. ASME International Mechanical Engineering Congress and Exposition Anaheim, USA: 01-708. https://doi.org/10.1115/imece2004-59309. 
13. Yi, Y. L.; Liu, P. P.; An, Z. J.; Jin, H. R. 2016. Tooth profile design and meshing characteristics analysis of any tooth difference pure rolling movable teeth transmission, Journal of Mechanical Engineering 52(11): 5056. https://doi.org/10.3901/jme.2016.11.050.

14. Mizukami, M.; Terada, H. 2009. Study on movable pins input type trochoid gear reducer (1st Report), Journal of the Japan Society for Precision Engineering 75(11): 1335-1339. https://doi.org/10.2493/jjspe.75.1335.

15. Mizukami, M.; Terada, H. 2010. Study on movable pins input type trochoid gear reducer (2nd Report), Journal of the Japan Society for Precision Engineering 76(6): 663-667. https://doi.org/10.2493/jjspe.76.663.

16.Robert, N. Design of machinery: an introduction to the synthesis and analysis of mechanisms and machines, Second Ed. WCB McGraw - Hill, USA. https://doi.org/10.5860/choice.30-1544.
Y. Yi, Y. Gao, L. He, H. Jin

\section{STUDY ON SWING MOVABLE TEETH DRIVE WITH EXTERNAL GENERATING WAVE MODE}

\section{S u m m a r y}

An external generating wave swing movable teeth drive based on sinusoidal acceleration and quintic polynomial motion has been newly developed. This drive consists of a wave generator as the input cam, a train of movable rollers which are uniformly distributed on the fixed separator, and a ring gear as the output cam. The teeth difference between the ring gear and the rollers is two. The input and output cam are engaged with the train of rollers simultaneously. The drive thus has the advantages of large gear ratio, compact structure, high torsional rigid and steady rotation, can be integrated with pulley drive to form a pulley reducer. In this paper, the motion principle is studied and the tooth profiles are derived. On this foundation, curvature of the ring gear and pressure angles of the drive are conducted. And kinematic simulation is also carried out. A physical prototype is manufactured to demonstrate the feasibility and correctness of the theoretical analysis model of the drive. This drive can be integrated with pulley drive to form a pulley reducer, which is promising for the fields needing compact structure and large transmission ratio.

Keywords: External generating wave, Movable teeth drive, Tooth profile, Kinematic simulation.

Received September 03, 2018

Accepted June 14, 2019 